\title{
Investigation of Nucleotide Diversity Based on 17 Sea Cucumber Mitochondrial Genomes and Assessment of Sea Cucumber Mitochondrial Gene Markers
}

\author{
Jongsun Park ${ }^{1,2^{*}}$ and Hong Xi \\ ${ }^{1}$ InfoBoss Inc, 301 room, 670, Seolleung-ro, Gangnam-gu, Seoul, Korea \\ ${ }^{2}$ InfoBoss Research Center, 301 room, 670, Seolleung-ro, Gangnam-gu, Seoul, Korea
}

*Corresponding author: Jongsun Park, InfoBoss Inc, 301 room, 670, Seolleung-ro,

Gangnam-gu, Seoul, Korea

Received Date: July 9, 2021

Published Date: July 27, 2021

\begin{abstract}
Apostichopus japonicus (Selenka, 1867), is one of the economically important species cultivated in the adjacent seas in East Asian countries. Due to this importance, 17 complete mitochondrial genomes of $A$. japonicus are available now. Nucleotide diversity of 17 A. japonicus mitochondrial genomes exhibited gene-specific features, which can be utilized to develop mitochondrial molecular marker sequences for this species. Together with 191 A. japonicus mitochondrial sequences, nucleotide diversities calculated from only mitochondrial genomes and all available sequences present different patterns, which may reflect the different coverage of samples. These investigations of nucleotide diversity can be applied for understanding intraspecific characteristics of mitochondrial genomes of certain species in near future.
\end{abstract}

Keywords: Nucleotide diversity, Mitochondrial genome, mitochondrial gene sequences, Apostichopus japonicus, Sea cucumber

\section{Introduction}

The sea cucumber, Apostichopus japonicus (Selenka, 1867), is one of the economically important species cultivated in the adjacent seas in East Asian countries, including China [1,2] and Korea $[3,4]$. It has been used as a source of nutritional foods as well as traditional medical resources widely [5,6]. Due to the importance of this species together with the benefits of next-generation sequencing (NGS) technologies [7-9], various genomics studies of $A$. japonicus have been conducted and published in recent 10 years: three whole genome sequence projects [10-12], ten complete mitochondrial genomes [13-16], as well as various transcriptome studies [17-29]. This amount of sequence resources is much larger than the other species except model organisms, such as Arabidopsis thaliana and Drosophila melanogaster, because the cost of generating NGS raw reads of multiple individuals is relatively high enough.
Mitochondrial genome sequences have been utilized for understanding phylogenetic relationships [30-33] as well as distinguishing population structures [34-41] because invertebrate mitochondrial genomes have a well-conserved structure [42] and keep a high copy number in the cell [43], which is favorable for assembling the mitochondrial genome from NGS raw reads. In addition, a short length of the mitochondrial genome reduces the costs to investigate intraspecific variations in the mitochondrial genomes [33-36,3840,44-56]. The current available mitochondrial genomes of $A$. japonicus are also enough to investigate its intraspecific characteristics in detail; however, there is no systematic bioinformatic analysis of all available A. japonicus mitochondrial genomes till now.

Here, we presented the investigation of nucleotide diversity of the 17 A.japonicus mitochondrial genomes to understand intraspe- 
cific characteristics of $A$. japonicus mitochondrial genomes in detail The overall trend of nucleotide diversity on A. japonicus mitochondrial genomes was investigated with displaying average nucleotide diversity of $A$. japonicus was higher than other species. Moreover COX1 and 16S rRNA sequences were investigated in detail with the 191 available mitochondrial marker sequences, displaying those different trends of nucleotide diversities between those originated from mitochondrial genomes and marker sequences were identified. Our investigation strategy can be expanded for further understanding of $A$. japonicus mitochondrial genomes, such as the relationship between nucleotide diversity and phenotypic variations, such as body colors [57], in near future.

\section{Materials and Methods}

\section{Preparation of mitochondrial genome of A. japonicus}

All seventeen mitochondrial genomes of $A$. japonicus used in this study were downloaded from the NCBI Nucleotide database and deposited into the GenomeArchive ${ }^{\circledR}[58]$ for conducting nucleotide diversity analyses. $16 \mathrm{~S}$ ribosomal RNAs and COX1 gene sequences were downloaded from the NCBI Nucleotide database.

\section{Nucleotide diversity analysis based on the A. japonicus mitochondrial genomes}

Multiple sequence alignment of 17 A. japonicus mitochondrial genomes (Table 1) was conducted using MAFFT v7.450 [59]. Based on this alignment, nucleotide diversity was calculated based on the method proposed by Nei and Li [60] using the perl script, which has been utilized in the previous studies [33, 61-63]. Nucleotide diversity of complete mitochondrial genomes was calculated with 500-bp windows with 200-bp step size. COX1 gene and 16S rRNA sequences were also aligned using MAFFT v7.450 [59]. Multiple sequence alignments were checked using Geneious Prime® v2020.2.4 (Biomatters Ltd., Auckland, New Zealand) because these sequences were amplified with different primers. Those of COXI genes and 16S rRNAs were also calculated with 100-bp windows with 50-bp step size due to their short length in comparison to the complete mitochondrial genomes. All bioinformatic analyses were conducted under the environment of the Genome Information System (GeIS ${ }^{\mathrm{TM}}$; http://geis.infoboss.co.kr/), which has been utilized in various genomic studies [44-53, 55, 56, 63-74].

Table 1: List of 17 A. japonicus mitochondrial genomes used in this study.

\begin{tabular}{|c|c|c|c|c|c|c|}
\hline No & Strain & Location & NCBI accession & Length (bp) & GC ratio (\%) & Ref. \\
\hline 1 & GreenQ & Qingdao, China & NC_012616 & 16,099 & 38 & [14] \\
\hline 2 & Red01 & Oita, Japan & GU557147 & 16,103 & 38.1 & [14] \\
\hline 3 & RedO2 & Oita, Japan & GU557148 & 16,103 & 38.1 & [14] \\
\hline 4 & RedK & Korea & FJ594963 & 16,107 & 38.1 & Unpub. \\
\hline 5 & GreenK & Korea & FJ594968 & 16,102 & 38.1 & Unpub. \\
\hline 6 & BlackK & Korea & FJ594967 & 16,108 & 38.1 & Unpub. \\
\hline 7 & GreenW & Weihai, China & EU294194 & 16,096 & 38.1 & [15] \\
\hline 8 & RedY & Yamaguchi, Japan & AB525761 & 16,103 & 38.1 & Unpub. \\
\hline 9 & GreenY & Yamaguchi, Japan & AB525760 & 16,106 & 38 & Unpub. \\
\hline 10 & BlackY & Yamaguchi, Japan & AB525437 & 16,105 & 38.1 & Unpub. \\
\hline 11 & GreenD & Dalian, China & FJ986223 & 16,109 & 38.1 & Unpub. \\
\hline 12 & BlackK2 & Geomun-do, Korea & MK216560 & 16,096 & 38.1 & [13] \\
\hline 13 & RedK2 & Geomun-do, Korea & MK216561 & 16,107 & 38.1 & [13] \\
\hline 14 & GreenK2 & Geomun-do, Korea & MK208925 & 16,106 & 38 & [13] \\
\hline 15 & RedR & Vladivostok, Russia & KP170616 & 16,100 & 38 & [16] \\
\hline 16 & GreenL & Lianyungang, China & KP170617 & 16,106 & 38 & [16] \\
\hline 17 & GreenW & Weihai, China & KP170618 & 16,106 & 38 & [16] \\
\hline
\end{tabular}

\section{Results and Discussions}

Till now, the seventeen $A$. japonicus mitochondrial genomes were sequenced from the three-color variants, red, green, and black sea cucumbers (Table 1) and the samples for mitochondrial genomes were captured in various regions of China, Korea, Japan, and Russia (Table 1). Their lengths range from 16,096 bp (MK216560) to $16,109 \mathrm{bp}$ (FJ986223), of which the difference is only $14 \mathrm{bp}$ $(0.087 \%$ against the longest mitochondrial genome). In the order Aspidochirotida including A. japonicus, two Holothuria species, Ho- lothuria leucospilota and Holothuria spinifera, contain more than one mitochondrial genome, displaying that intraspecific differences of the three H. leucosilota mitochondrial genomes (GenBank accessions are MK801674, NC_046849, and MN276190) [75, 76] and two H. spinifera mitochondrial genomes (GenBank accessions are MW218896 and NC_046508) [77] are 67 bp and 23 bp, respectively. It indicates that the intraspecific length difference of $A$. japonicus mitochondrial genome is relatively small. In addition, This intraspecific difference is also smaller than those of several insect spe- 
cies, Sogatella furcifera (43 bp from three mitochondrial genomes; Park et al., in submission) [78], Laodelphax striatellus (155 bp from eighty-seven mitochondrial genomes) [34, 35, 37], and Aphis gossypii (186 bp from five mitochondrial genomes) [46, 48, 52, 55, 56] except that of Nilaparvata lugens $(14 \mathrm{bp}$ from the five mitochondrial genomes excluding them without control regions) [38-40, 79] (Park et al., in submission). It implies that intraspecific differences of $A$. japonica mitochondrial genome with considering the geographical distribution of the seventeen samples are not so high.

GC ratio of the seventeen $A$. japonicus mitochondrial genome ranges from $38.0 \%$ to $38.1 \%$ (Table 1 ). There are no differences in the number of genes and gene orders among the seventeen mitochondrial genomes, displaying the conserved manner of mitochondrial genomes, which is also same to those of S. furcifera, L. striatellus, A. gossypii, and N. lugens.

Sequence-level intraspecific variations are also important, especially for distinguish the origin of samples. One useful way to investigate intraspecific variations is nucleotide diversity. Nucleotide diversity of 17 A. japonicus displays the highest peak in the control regions and the lowest in 16S ribosomal RNA (rRNA) and 12S rRNA (Figure 1). Usually, control regions display high level of nucleotide diversity [33]. Average nucleotide diversity was calculated as 0.00374 , which is much larger than those of nine $S$. furcifera mitochondrial genomes (0.000246; Choi et al., in preparation; Park et al in preparation) [78] and L. striatellus (0.00188) [34, 35, 37], indicating that there is relatively high level of intraspecific variations on A. japonica mitochondrial genomes.

In detail, the 3' regions of COX1, COX2, and COX3 and the 5' regions of CYTB and ND1 display the lower nucleotide diversity of the average nucleotide diversity (Figure 1), indicating that these regions are conserved on A. japonicus mitochondrial genomes. In addition, tRNA cluster between the control region and ND1 gene also present lower nucleotide diversity than the average (Figure 1). In addition, nucleotide diversity of COX1 gene displays the differences between 5' region and 3' region: 5' region of COX1 shows higher nucleotide diversity than average, while the 3 ' region presents lower (Figure 1). It indicates that the resolution of molecular marker based on COX1 gene will be different along with the region of COX1.

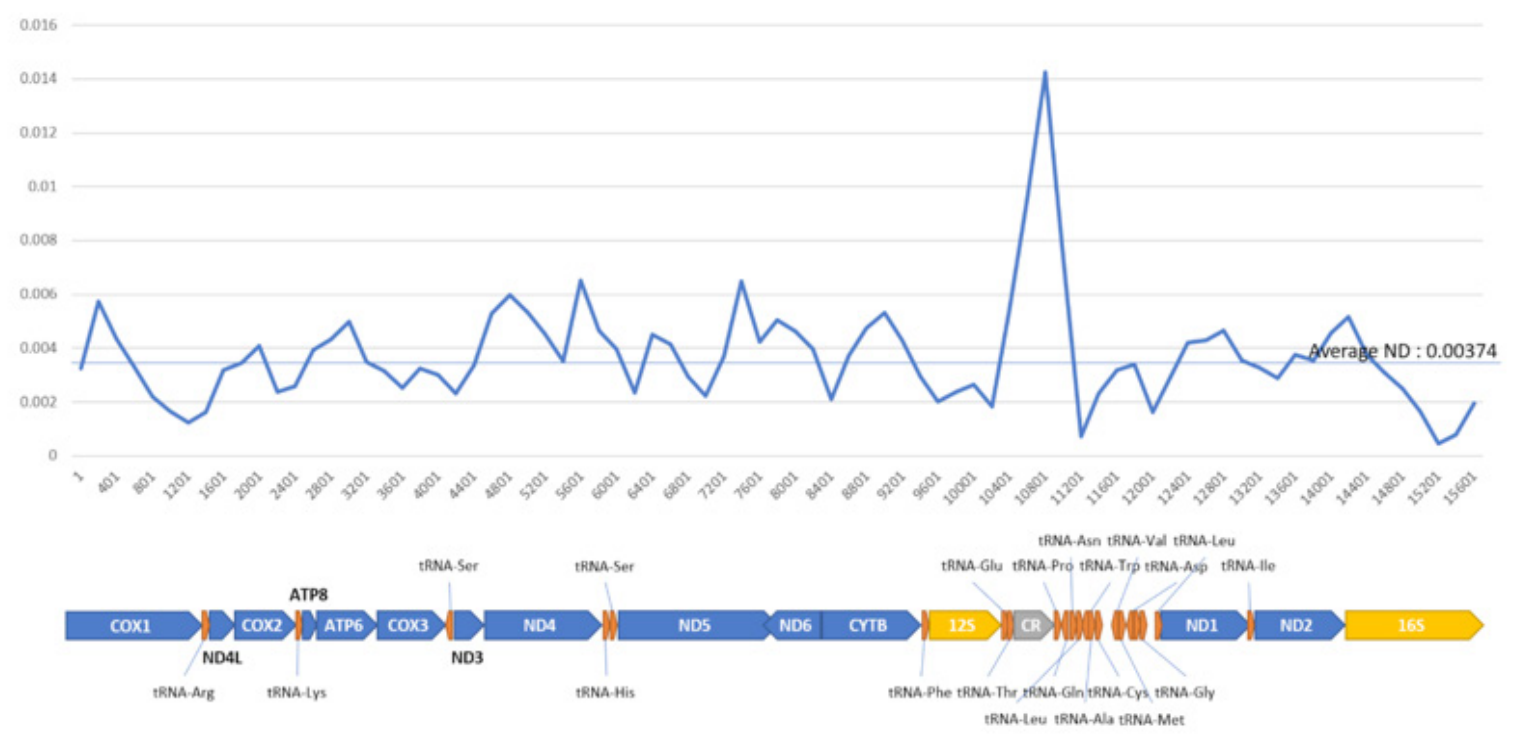

Figure 1: Nucleotide diversity of $17 \mathrm{~A}$. japonicus mitochondrial genome.

The $\mathrm{x}$-axis indicates nucleotide position of multiple sequence alignment of $17 \mathrm{~A}$. japonicus mitochondrial genomes. Y-axis presents nucleotide diversity. The thin blue line on the graph indicates average nucleotide diversity. Below the graph, 37 genes were drawn with colored arrow diagrams: blue arrows indicate protein coding-genes, orange arrows indicate transfer RNAs, yellow arrows mean ribosomal RNAs, and grey arrows show control region.

Currently available 191 mitochondrial molecular marker sequences of $A$. japonicus in NCBI consist of $9616 \mathrm{~S}$ ribosomal RNA and 95 COX1 genes. Three out of 95 COX1 genes contain 16S rRNA sequences so that these three COX1 genes cover 5 ' region. The remaining COX1 genes also cover 5 ' region but after the coverage of the three genes. Nucleotide diversity of COX1 genes together with 17 complete COX1 genes originated from the complete mitochondrial genomes display that the highest peak of nucleotide diversity is 0.013 at 351 to $450 \mathrm{bp}$ region of the COX1 gene; while 17 COX1 genes from the mitochondrial genome show the valley point, show- ing opposite trend (Figure 2A). Moreover, the region between 451 bp to 651 bp shows the reverse manner: 17 COX1 genes from the mitochondrial genome show a high nucleotide diversity than that of all COX1 genes (Figure 2A). It indicates that the coverage of the samples used in complete mitochondrial genomes is different from that of all COX1 genes as well as the $5^{\prime}$ region of COX1 provides enough resolution to conduct phylogeny studies.

Nucleotide diversity of 16S rRNA sequences show a much higher value than that of $16 \mathrm{~S}$ rRNA from 17 mitochondrial genomes 
(Figure 2B). The region of which nucleotide diversity is high (601 bp to 1301 bp region in Figure 2B) was sequenced in most of $16 \mathrm{~S}$ rRNA marker sequences. It is interesting that the average nucleo- tide diversity of 16S rRNA (0.04691) is higher than that of COX1 markers (0.007155), suggesting that rRNA sequences may have better resolution than COX1 in A. japonicus.
(A)

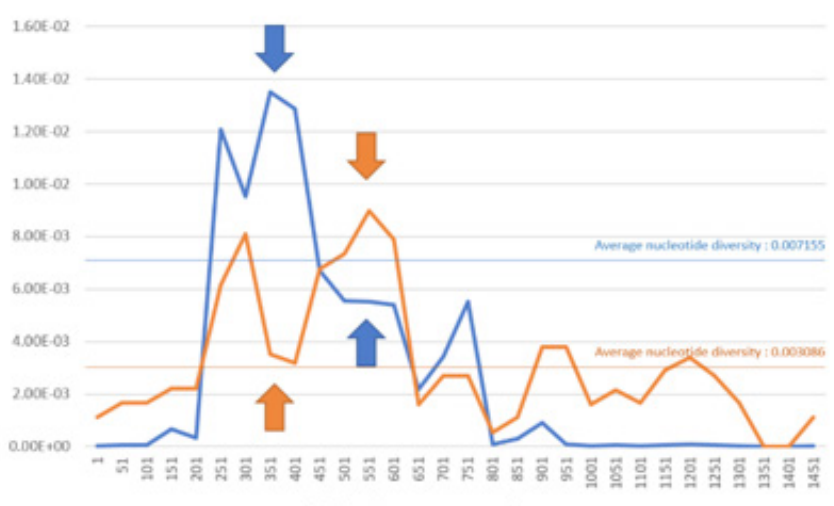

(B)

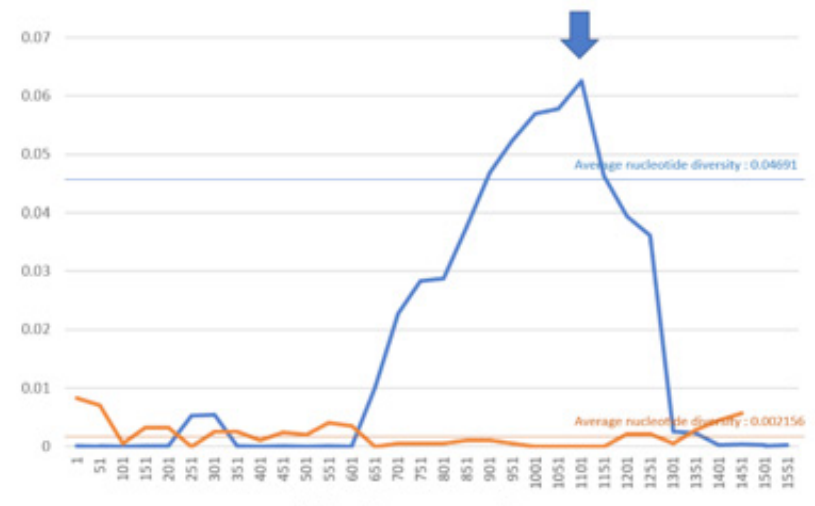

Figure 2: Nucleotide diversities of COX1 and 16S rRNA sequences of $A$. japonicus.

(A) Nucleotide diversity of COX1 genes from mitochondrial sequences and complete mitochondrial genomes of $A$. japonicus. (B) Nucleotide diversity of $16 \mathrm{~S}$ rRNAs from mitochondrial sequences and complete mitochondrial genomes of $A$. japonicus. In both panels, the X-axis means nucleotide position of multiple sequence alignments of both genes. Blue lines mean nucleotide diversity calculated from all mitochondrial genes and genes extracted from complete mitochondrial genomes and orange lines indicate nucleotide diversity calculated only from 17 complete mitochondrial genomes. Blue and orange arrows indicate interesting points on nucleotide diversities.

Through this investigation of nucleotide diversity of $17 \mathrm{~A}$. japonicus mitochondrial genome, overall features of intraspecific variations of $A$. japonicus mitochondrial genomes and $16 \mathrm{~S}$ rRNA and COX1 marker sequences were identified. Even though the cost to obtain complete mitochondrial genomes using NGS technologies is still not cheap, enough mitochondrial genomes in certain species can provide more information of intraspecific characteristics. In near future, this method of investigation can be applied to the species which have more mitochondrial genome sequences for selecting the best marker sequences of that species as well as for understanding intraspecific characteristics of mitochondrial genomes.

\section{Funding}

This work was supported by both InfoBoss Research Grant (IBG-0020).

\section{Acknowledgment}

None.

\section{Conflict of Interest}

No conflict of interest.

\section{References}

1. Zhang C, Wang Y, Rong X, Sun H, Dong S (2004) Natural resources, culture and problems of sea cucumber worldwide. Marine Fisheries Research 25(3): 89-97.

2. Liu H, Zheng F, Sun X, Hong X, Dong S, et al. (2010) Identification of the pathogens associated with skin ulceration and peristome tumescence in cultured sea cucumbers Apostichopus japonicus (Selenka). J Invertebr Pathol 105(3): 236-242.

3. Park S, Hong S, Yoon I, Lovatelli A (2015) Apostichopus japonicus:“Dolgi Haesam" in the Republic of Korea (South Korea). Developments in Aquaculture and Fisheries Science Elsevier: 423-431.

4. Park J, An JH, Kim Y, Kim D, Yang BG, et al. (2020) Database of National Species List of Korea: the taxonomical systematics platform for managing scientific names of Korean native species. Journal of Species Research 9(3): 233-246.

5. Dang H, Zhang T, Yi F, Ye S, Liu J, et al. (2019) Enhancing the immune response in the sea cucumber Apostichopus japonicus by addition of Chinese herbs Houttuynia cordata Thunb as a food supplement. Aquaculture and Fisheries 4(3): 114-121.

6. Wu Yn, Liu P, Li Q Jiang C-j, Li H (2011) Effects of a compound Chinese herbal medicine on immunity and disease resistance in sea cucumber Apostichopus japonicus [J]. Journal of Dalian Ocean University : 4.

7. Metzker ML (2010) Sequencing technologies-the next generation. Nat Rev Genet 11(1): 31-46.

8. Goodwin S, McPherson JD, McCombie WR (2016) Coming of age: ten years of next-generation sequencing technologies. Nat Rev Genet 17(6): 333-351.

9. Bleidorn C (2016) Third generation sequencing: technology and its potential impact on evolutionary biodiversity research. Systematics and biodiversity 14(1): 1-8.

10. Jo J, Oh J, Lee HG, Hong HH, Lee SG, et al. (2017) Draft genome of the sea cucumber Apostichopus japonicus and genetic polymorphism among color variants. Gigascience 6(1): 1-6.

11. Zhang X, Sun L, Yuan J, Sun Y, Gao Y, et al. (2017) The sea cucumber genome provides insights into morphological evolution and visceral regeneration. PLoS Biology 15(10): e2003790. 
12. Li Y, Wang R, Xun X, Wang J, Bao L, et al. (2018) Sea cucumber genome provides insights into saponin biosynthesis and aestivation regulation. Cell discovery 4(1): 1-17.

13. Park J, Xi H, Park C (2019) The complete mitochondrial genomes from three body color variants of sea cucumbers, Apostichopus japonicus (Selenka, 1867). Mitochondrial DNA Part B 4(1): 836-837.

14. Sun XJ, Li Q Kong LF (2010) Comparative mitochondrial genomics within sea cucumber (Apostichopus japonicus): Provide new insights into relationships among color variants. Aquaculture 309(1-4): 280285.

15. Shen X, Tian M, Liu Z, Cheng H, Tan J, et al. (2009) Complete mitochondrial genome of the sea cucumber Apostichopus japonicus (Echinodermata: Holothuroidea): the first representative from the subclass Aspidochirotacea with the echinoderm ground pattern. Gene 439(1-2): 79-86.

16. Zhang W, Cao Z, Li Y, Zhao H, Huang J, et al. (2016) Taxonomic status of the three-color variants in sea cucumber (Apostichopus japonicus): evidence from mitochondrial phylogenomic analyses. Mitochondrial DNA A DNA Mapp Seq Anal 27(4): 2330-2333.

17. Du H, Bao Z, Hou R, Wang S, Su H, et al. (2012) Transcriptome sequencing and characterization for the sea cucumber Apostichopus japonicus (Selenka, 1867). PloS one 7(3): e33311.

18. Zhou Z, Dong Y, Sun H, Yang A, Chen Z, et al. (2014) Transcriptome sequencing of sea cucumber (Apostichopus japonicus) and the identification of gene-associated markers. Mol Ecology Resour 14(1): 127-138.

19. Li C, Fang H, Xu D (2019) Effect of seasonal high temperature on the immune response in Apostichopus japonicus by transcriptome analysis. Fish Shellfish Immunol 92: 765-771.

20. Zhang P, Li C, Zhu L, Su X, Li Y, et al. (2013) De novo assembly of the sea cucumber Apostichopus japonicus hemocytes transcriptome to identify miRNA targets associated with skin ulceration syndrome. PLoS One 8(9): e73506

21. Rowe ML, Achhala S, Elphick MR (2014) Neuropeptides and polypeptide hormones in echinoderms: new insights from analysis of the transcriptome of the sea cucumber Apostichopus japonicus. Gen Comp Endocrinol 197: 43-55.

22. Yang A, Zhou Z, Pan Y, Jiang J, Dong Y, et al. (2016) RNA sequencing analysis to capture the transcriptome landscape during skin ulceration syndrome progression in sea cucumber Apostichopus japonicus. BMC genomics 17: 459 .

23. Gao Q, Liao M, Wang Y, Li B, Zhang Z, et al. (2015) Transcriptome analysis and discovery of genes involved in immune pathways from coelomocytes of sea cucumber (Apostichopus japonicus) after vibrio splendidus challenge. Int J Mol Sci 16(7): 16347-16377.

24. Ding K, Zhang L, Sun L, Lin C, Feng Q et al. (2019) Transcriptome analysis provides insights into the molecular mechanisms responsible for evisceration behavior in the sea cucumber Apostichopus japonicus. Comparative Biochemistry and Physiology Part D: Genomics and Proteomics 30: 143-57.

25. Gao L, He C, Bao X, Tian M, Ma Z (2017) Transcriptome analysis of the sea cucumber (Apostichopus japonicus) with variation in individual growth. Plos One 12(7): e0181471.

26. Jo J, Park J, Lee HG, Kern EM, Cheon S, et al. (2016) Comparative transcriptome analysis of three-color variants of the sea cucumber Apostichopus japonicus. Marine genomics 28: 21-24.

27. Zhou X, Cui J, Liu S, Kong D, Sun H, et al. (2016) Comparative transcriptome analysis of papilla and skin in the sea cucumber, Apostichopus japonicus. Peer] 4: e1779.

28. Ma D, Yang H, Sun L, Xu D (2014) Comparative analysis of transcriptomes from albino and control sea cucumbers, Apostichopus japonicus. Acta Oceanologica Sinica 33(8): 55-61.
29. Gao K, Wang Z, Qiu X, Song J, Wang H, et al. (2019) Transcriptome analysis of body wall reveals growth difference between the largest and smallest individuals in the pure and hybrid populations of Apostichopus japonicus. Comparative Biochemistry and Physiology Part D: Genomics and Proteomics 31: 100591.

30. Park J, Xi H, Park J (2021) Complete mitochondrial genome of the acrobat ant Crematogaster teranishii Santschi, 1930 (Formicidae; Hymenoptera). Mitochondrial DNA Part B 6(2): 593-595.

31. Wang K, Li X, Ding S, Wang N, Mao M, et al. (2016) The complete mitochondrial genome of the Atylotus miser (Diptera: Tabanomorpha: Tabanidae), with mitochondrial genome phylogeny of lower Brachycera (Orthorrhapha). Gene 586(1):184-196.

32. Peng Z, Wang J, He S (2006) The complete mitochondrial genome of the helmet catfish Cranoglanis bouderius (Siluriformes: Cranoglanididae) and the phylogeny of otophysan fishes. Gene 376(2): 290-297.

33. Lee J, Park J, Xi H, Park J (2020) Comprehensive analyses of the complete mitochondrial genome of Figulus binodulus (Coleoptera: Lucanidae). J Insect Sci 20(5): 10 .

34. Seo BY, Jung JK, Ho Koh Y, Park J (2019) The complete mitochondrial genome of Laodelphax striatellus (Fallén,1826)(Hemiptera: Delphacidae) collected in a southern part of Korean peninsula. Mitochondrial DNA B Resour 4(2): 2242-2243.

35. Park J, Jung JK, Ho Koh Y, Park J, Seo BY (2019) The complete mitochondrial genome of Laodelphax striatellus (Fallén, 1826) (Hemiptera: Delphacidae) collected in a mid-Western part of Korean peninsula. Mitochondrial DNA B Resour 4(2): 2229-2230.

36. Kim B, Bae Y, Lee J, Park J, Choi YS, et al. (2021) The complete mitochondrial genome of the far Eastern myotis: Myotis bombinus Thomas, 1906 in mainland of Korea (Chiroptera, Vespertilionidae). Mitochondrial DNA B Resour 6(2): 615-616.

37. Sun JT, Duan XZ, Hoffmann AA, Liu Y, Garvin MR, et al. (2019) Mitochondrial variation in small brown planthoppers linked to multiple traits and probably reflecting a complex evolutionary trajectory. Molecular ecology 28(14): 3306-3323.

38. Choi NJ, Lee BC, Park J, Park J (2019) The complete mitochondrial genome of Nilaparvata lugens (Stål, 1854) captured in China (Hemiptera: Delphacidae): investigation of intraspecies variations between countries. Mitochondrial DNA Part B 4(1): 1677-1678.

39. Park J, Kwon W, Park J, Kim HJ, Lee BC, et al. (2019) The complete mitochondrial genome of Nilaparvata lugens (stål, 1854) captured in Korea (Hemiptera: Delphacidae). Mitochondrial DNA Part B 4(1): $1674-$ 1676.

40. Choi NJ, Lee BC, Park J, Park J (2020) The complete mitochondrial genome of Nilaparvata lugens (Stål, 1854) captured in Guangxi province, China (Hemiptera: Delphacidae): identification of the origin of $N$. lugens migrated to Korea. Mitochondrial DNA Part B 5(2): 1960-1961.

41. Park J, Jung JK, Lee GS, Cho J, Koh YH (2019) Comparative analyses of Laodelphax striatellus mitochondrial genomes: Insight haplotypes of mitogenomes in three East Asian Countries. Fall International Conference of KSAE Seoul, Korea.

42. Lavrov DV, Bell E (2014) Mitochondrial genomes in invertebrate animals. Molecular life sciences: an encyclopedic reference New York (NY): Springer New York: 1-8.

43. Trinei M, Berniakovich I, Pelicci PG, Giorgio M (2006) Mitochondrial DNA copy number is regulated by cellular proliferation: A role for Ras and p66Shc. Biochim Biophys Acta 1757(5-6): 624-630.

44. Choi NJ, Xi H, Park J (2021) A Comparative Analyses of the Complete Mitochondrial Genomes of Fungal Endosymbionts in Sogatella furcifera, White-Backed Planthoppers. Int J Genomics 2021: 6652508.

45. Lee B, Park J (2021) The complete chloroplast genome of Zoysia matrella (L.) Merr. isolated in Korea (Poaceae): investigation of intraspecific variations on chloroplast genomes. Mitochondrial DNA B Resour 6(2): 572-574. 
46. Niu R, Gao X, Luo J, Wang L, Zhang K, et al. (2021) Mitochondrial genome of Aphis gossypii Glover cucumber biotype (Hemiptera: Aphididae). Mitochondrial DNA B Resour 6(3): 922-924.

47. Park J, Kim Y, Kwon W, Xi H, Park CH (2021) The complete chloroplast genome sequence of new species candidate of Plantago depressa Willd in Korea (Plantaginaceae). Mitochondrial DNA Part B Resources 6(7): 1961-1963

48. Park J, Lee J, Lee W (2021) The complete mitochondrial genome of Aphis gossypii Glover, 1877 (Hemiptera: Aphididae) isolated from Leonurus japonicus in Korea. Mitochondrial DNA B Resour 6(1): 62-65.

49. Park J, Park S, Jang T, Kim G, Park JH (2021) The complete chloroplas genome of Abeliophyllum distichum $f$. lilacinum Nakai (Oleaceae) from the Chungbuk Province, Korea. Mitochondrial DNA B Resour 6(6): 1754 1756 .

50. Park J, Xi H, Kim Y (2020) The complete chloroplast genome of Arabidopsis thaliana isolated in Korea (Brassicaceae): an investigation of intraspecific variations of the chloroplast genome of Korean A. Thaliana. Int J Genomics 2020: 3236461.

51. Park J, Xi H, Kim Y (2021) The complete mitochondrial genome of Arabidopsis thaliana (Brassicaceae) isolated in Korea. Korean Journal of Plant Taxonomy 51(2): 176-180.

52. Park J, Xi H, Kim Y, Park J, Lee W (2019) The complete mitochondrial genome of Aphis gossypii Glover, 1877 (Hemiptera: Aphididae) collected in Korean peninsula. Mitochondrial DNA B Resour 4(2): 3007-3009.

53. Yoo SC, Oh Sh, Park J (2021) Phylogenetic position of Daphne genkwa (Thymelaeaceae) inferred from complete chloroplast data. Korean J Pl Taxon 51(2): 171-175.

54. Min J, Kim Y, Xi H, Jang T, Kim G, et al. (2019) The complete chloroplast genome of a new candidate cultivar, Sang Jae, of Abeliophyllum distichum Nakai (Oleaceae): initial step of A. distichum intraspecies variations atlas. Mitochondrial DNA B Resour 4(2): 3716-3718.

55. Bae Y, Park J, Lee W (2020) The complete mitochondrial genome of Aphis gossypii Glover, 1877 (Hemiptera: Aphididae) isolated from Plantago asiatica in Korea. Mitochondrial DNA B Resour 5(3): 2878-2880.

56. Zhang S, Luo J, Wang C, Lv L, Li C, et al. (2016) Complete mitochondrial genome of Aphis gossypii Glover (Hemiptera: Aphididae). Mitochondrial DNA Part A 27(2): 854-855.

57. Yang H, Jean Francois Hamel, Annie Mercier (2015) The sea cucumber Apostichopus japonicus: history, biology and aquaculture: Academic Press.

58. Park J, Xi H (2018) Genome Archive (R): standardized genome repository for supporting large-scale genome analyses. Plant and Animal Genome XXVI Conference: PAG.

59. Katoh K, Standley DM (2013) MAFFT multiple sequence alignment software version 7: improvements in performance and usability. Mol Biol Evol 30(4): 772-780.

60. Nei M, Li WH (1979) Mathematical model for studying genetic variation in terms of restriction endonucleases. Proceedings of the National Academy of Sciences 76(10): 5269-5273.

61. Kim Y, Park J, Chung Y (2019) Comparative Analysis of Chloroplast Genome of Dysphania ambrosioides (L.) Mosyakin \& Clemants Understanding Phylogenetic Relationship in Genus Dysphania $\mathrm{R}$. Br. Korean Journal of Plant Resources 32(6): 644-668.

62. Park J, Xi H, Oh SH (2020) Comparative chloroplast genomics and phylogenetic analysis of the Viburnum dilatatum complex (Adoxaceae) in Korea. Korean Journal of Plant Taxonomy 50(1): 8-16.

63. Park J, Min J, Kim Y, Chung Y (2021) The Comparative Analyses of Six Complete Chloroplast Genomes of Morphologically Diverse Chenopodium album L. (Amaranthaceae) Collected in Korea. Int J Genomics 2021 6643444 .
64. Joo S, Lee J, Lee DY, Xi H, Park J (2020) The complete mitochondrial genome of the millipede Epanerchodus koreanus Verhoeff, 1937 collected in limestone cave of Korea (Polydesmidae: Polydesmida). Mitochondrial DNA B Resour 5(4): 3845-3847.

65. Park J, Kwon W, Hong SB, Han KH (2020) First record of the complete mitochondrial genome of a saprotrophic and opportunistic human pathogenic Fungus, Scopulariopsis brevicaulis. Mycobiology 48(6): 528531.

66. Park J, Kim Y, Kwon W, Xi H, Park J (2019) The complete mitochondrial genome of Neocaridina heteropoda koreana Kubo, 1938 (Decapoda: Atyidae). Mitochondrial DNA B Resour 4(2): 2332-2334

67. Baek J, Park S, Lee J, Min J, Park J, et al. (2021) The complete chloroplast genome of Chrysanthemum zawadskii Herbich (Asteraceae) isolated in Korea. Mitochondrial DNA B Resour 6(7): 1956-1958.

68. Suh HJ, Min J, Park J, Oh SH (2021) The complete chloroplast genome of Aruncus dioicus var. kamtschaticus (Rosaceae). Mitochondrial DNA B Resour 6(3): 1256-1258.

69. Park J, Park CH, Park J (2021) Complete mitochondrial genome of the H3 haplotype Argentine ant Linepithema humile (Mayr, 1868)(Formicidae; Hymenoptera). Mitochondrial DNA B Resour 6(3): 786-788.

70. Park J, Lee SH, Kim JH (2021) Complete genome sequence of the endosymbiotic bacterium "Candidatus Riesia pediculicola". Microbiol Resour Announc 10(18): e01181-20.

71. Park J, Xi H, Kim Y, Kim M (2021) Complete Genome Sequence of Lentilactobacillus parabuchneri Strain KEM. Microbiol Resour Announc 10(20): e01208-20.

72. Park J, Xi H, Park J, Nam SJ, Lee YD (2020) Complete genome sequence of the Blochmannia endosymbiont of Camponotus nipponensis. Microbiol Resour Announc 9(29): e00703-20.

73. Park J, Xi H, Park J, Lee W (2020) The complete mitochondrial genome of fungal endosymbiont, Ophiocordycipitaceae sp., isolated from Ricania speculum (Hemiptera: Ricaniidae). Mitochondrial DNA Part B 5(2): 1888-1889.

74. Park J, Xi H, Park J (2021) Complete genome sequence of a Blochmannia endosymbiont of Colobopsis nipponica. Microbiol Resour Announc 10(17).

75. Yang Q, Lin Q, Wu J, Tran NT, Huang R, et al. (2019) Complete mitochondrial genome of Holothuria leucospilata (Holothuroidea, Holothuriidae) and phylogenetic analysis. Mitochondrial DNA B Resour 4(2): 2751-2752

76. Zhong S, Liu Y, Huang L, Zhao Y, Huang G (2019) The complete mitochondrial genome of black sea cucumber Holothuria leucospilota (Aspidochirotida holothuriidae). Mitochondrial DNA B Resour 4(2): 3377-3378.

77. Ding B, Sun Y, Rong F, Guo C, Wang Y, et al. (2020) The complete mitochondrial genome of Holothuria spinifera (Théel, 1866). Mitochondrial DNA B Resour 5(2): 1679-1680.

78. Zhang KJ, Zhu WC, Rong X, Liu J, Ding XL, et al. (2014) The complete mitochondrial genome sequence of Sogatella furcifera (Horváth) and a comparative mitogenomic analysis of three predominant rice planthoppers. Gene 533(1): 100-109.

79. Zhang KJ, Zhu WC, Rong X, Zhang YK, Ding XL, et al. (2013) The complete mitochondrial genomes of two rice planthoppers, Nilaparvata lugens and Laodelphax striatellus: conserved genome rearrangement in Delphacidae and discovery of new characteristics of atp8 and tRNA genes. BMC Genomics 14: 417. 